\title{
Second line immune checkpoint inhibition in urothelial cancer
}

\author{
Kilian M. Gust ${ }^{1}$, Mohammad Abufaraj ${ }^{2}$, David D’Andrea ${ }^{1}$, Marco Moschini $^{3}$, Francesco Soria ${ }^{4}$, \\ Shahrokh F. Shariat ${ }^{1,5,6,7,8}$
}

\begin{abstract}
${ }^{1}$ Department of Urology, Comprehensive Cancer Center, Medical University of Vienna, Vienna, Austria; ${ }^{2}$ Department of Special Surgery, University of Jordan, Amman, Jordan; ${ }^{3}$ Department of Urology, Luzerner Kantonsspital, Lucerne, Switzerland; ${ }^{4}$ Division of Urology, Azienda Ospedaliera Città della Salute e della Scienza di Torino, Torino, Italy; ${ }^{5}$ Department of Urology, Weill Cornell Medical College, New York, NY, USA; ${ }^{6}$ Department of Urology, University of Texas Southwestern, Dallas, TX, USA; ${ }^{7}$ Department of Urology, Second Faculty of Medicine, Charles University, Prague, Czech Republic; ${ }^{8}$ Institute for Urology and Reproductive Health, I.M. Sechenov First Moscow State Medical University, Moscow, Russia

Correspondence to: Kilian M. Gust, MD, FEBU. Department of Urology and Comprehensive Cancer Center, Medical University of Vienna, Vienna General Hospital, Waehringer Guertel 18-20, Vienna, Austria. Email: kilian.gust@meduniwien.ac.at.

Comment on: Fradet Y, Bellmunt J, Vaughn DJ, et al. Randomized phase III KEYNOTE-045 trial of pembrolizumab versus paclitaxel, docetaxel, or vinflunine in recurrent advanced urothelial cancer: results of $>2$ years of follow-up. Ann Oncol 2019. [Epub ahead of print].
\end{abstract}

Submitted Aug 25, 2019. Accepted for publication Sep 10, 2019.

doi: $10.21037 /$ tau.2019.09.18

View this article at: http://dx.doi.org/10.21037/tau.2019.09.18

Urothelial cancer (UC) is devastating disease, once it reaches muscle invasive (MIBC) and metastatic stage. Even when performing radical cystectomy (RC) with pelvic lymphadenectomy in patients with MIBC, approximately half of the patients will develop metastasis and still no longterm cure for metastatic urothelial cancer (mUC) has been found (1). Neoadjuvant cisplatin-based chemotherapy is considered a standard approach in pT2-pT4a pN0 disease which can improve survival outcomes after surgery. Though, it is still underutilized. Platinum-based combination chemotherapies are also used to treat mUC in first line $(2,3)$. Till 2016, the only approved second line drug for patients who failed during or after first line platinum-based chemotherapy was vinflunine. With limited treatment effect in a phase 3 trial compared to best supportive care (BSC), it has been approved in Europe only $(4,5)$. In the US, taxane based chemotherapies were commonly used in this setting.

Prior to approval of immune checkpoint inhibitors (CPI), the median overall survival (mOS) for metastatic UC was poor with around 15 months using first line cisplatin-based combination chemotherapies $(2,3)$ and roughly 10 months for patients being ineligible for cisplatin, and therefore receiving carboplatin-based combination chemotherapy (6). Patients receiving second line chemotherapy after platinumbased regimens reached a mOS of about 7 months (5).

With approval of the first CPI atezolizumab in 2016 in the second line setting, the treatment of mUC has changed dramatically (7). Although, response rates in unselected patient population are still not high, all substances have in common, that patients who respond to CPI treatment seem to have long-lasting responses, with the median duration of response (DoR) not being reached in most of trials (8-15). Several other targeting agents [i.e., erdafitinib (16), enfortumab vedotin (17)] are under evaluation in clinical trials and have shown promising results, that hopefully will enlarge treatment options in $\mathrm{mUC}$ and potentially in the neoadjuvant setting (18-20).

In first line setting, gemcitabine/cisplatin is still standard for metastatic and locally advanced, non-resectable mUC. Though, nearly half of patients are ineligible for cisplatinbased chemotherapy, which is commonly defined by the criteria proposed by Galsky et al. (21). For cisplatinineligible patients atezolizumab (9) and pembrolizumab (8) have been approved by the European Medicines Agency (EMA) and the North-American Food and Drug Administration (FDA) for patients with $\mathrm{PD}-\mathrm{L} 1$ positive tumors, defined by the presence of PD-L1 positively stained cells and analyzed by either tumor infiltrating immune cells (IC) $\geq 5 \%$ or the combined positive score (CPS) $\geq 10(22,23)$.

In second line, CPI have revolutionized the treatment mUC. Because of urgent medical need all substances were initially approved based on early phase I/II data independent of the PD-L1 status. In Europe, three substances are approved for treatment of mUC after failure of platinum- 
based chemotherapy [pembrolizumab (10), atezolizumab $(13,24)$, and nivolumab $(15)]$. In addition, durvalumab (14) and avelumab (12) are approved in this indication in the US only.

Though, pembrolizumab is the only CPI with proven level 1 evidence from KEYNOTE-045 being superior to second line chemotherapy for mUC (10). In this trial, a total of 542 patients were randomly assigned to receive either pembrolizumab or investigators' choice of second line chemotherapy (vinflunine, docetaxel or paclitaxel). In the initial NEFM publication, Bellmunt et al. presented a beneficial mOS of 10.3 [95\% confidence interval (CI), 8.0 11.8 months in the pembrolizumab group, as compared to 7.4 (95\% CI: 6.1-8.3) months in the chemotherapy group [hazard ratio (HR) for death, 0.73 ; $95 \%$ CI: 0.59 to 0.91 ; $\mathrm{P}=0.002]$. In the total population, the objective response rate (ORR) was significantly higher in the pembrolizumab group $(21.1 \% ; 95 \%$ CI: $16.4-26.5)$ than in the chemotherapy group (11.4\%; 95\% CI: 7.9-15.8; $\mathrm{P}=0.001)$. Pembrolizumab showed less treatment-related events of grade $3-5$ than second line chemotherapy $(15.0 \%$ vs. $49.4 \%$ of patients), which resulted in lower rate of treatmentrelated discontinuation of therapy (5.6\% vs. 11.0\%) (10).

Recently, Fradet et al. published updated results of this trial with a $>2$ years follow up (11). The mOS is nearly unchanged with 10.1 months for the pembrolizumab group as compared to 7.3 months for patients treated with chemotherapy (HR 0.70, 95\% CI: 0.57-0.85; $\mathrm{P}=0.00015$ ). Interestingly, no statistically significant difference in progression free survival (PFS) between the pembrolizumab and chemotherapy arms has been observed (2.1 months; 95\% CI: $2.0-2.2$ vs. 3.3 months; 95\% CI: 2.4-3.6; HR 0.96; 95\% CI: 0.79-1.16). However, the 2-yr PFS rates were higher in pembrolizumab treated patients (12.4\%) than in the group treated with chemotherapy (3.0\%) (11). The median DoR to treatment in patients responded to chemotherapy $(\mathrm{n}=30)$ is only 4.4 months (range, 1.4+ to $29.9+$ months), while is still has not been reached for patients initially responded to pembrolizumab $(1.6+$ to $30.0+)$ (11). After a median follow-up of 27.7 months, this translates into one-year survival rates of $44.2 \%$ vs. $19.8 \%$ and two-year survival rates of $26.9 \%$ vs. $14.3 \%$ for patients treated with pembrolizumab vs. second line chemotherapy, respectively. Remarkably, the OS rate of $14.3 \%$ at 2 years for the chemotherapy arm, which hasn't been observed in previous second line clinical trials, could possibly be explained by the fact, that the majority of patients alive at 24 months (60.6\%), received a third line CPI treatment (11).
In terms of safety, treatment-related adverse events (AEs) occurred less frequently in patients receiving pembrolizumab $(62.0 \%)$ than in chemotherapy treated patients $(90.6 \%)$. The most common AEs were pruritus for the pembrolizumab arm and alopecia, fatigue, anemia, nausea, constipation, decreased appetite, and neutropenia for the chemotherapy arm (11). The longer follow-up confirmed data from the initial publication, underlining the safety and better tolerability of pembrolizumab compared to chemotherapy in this therapeutic setting.

Atezolizumab is the other CPI that has been evaluated in a second line, phase III clinical trial (IMvigor211) (13). The phase II trial IMvigor210 (cohort 2) showed promising results, with an impressive mOS of 11.4 months in patients with PD-L1 positive tumors (IC2/3), based on staining with the antibody SP142 and analysis of tumor infiltrating immune cells (IC $\geq 5 \%$ ). Patients with low PD-L1 (IC1: $\geq 1 \%$ and $<5 \%$ ) or no PD-L1 (IC0: $<1 \%$ ) expression had significantly worse outcome with a mOS of 6.7 (95\% CI: 5.1-8.8) and 6.5 (95\% CI: 4.4-8.3) months, respectively (24).

Though, the following IMvigor211 trial failed to proof superiority to second line chemotherapy (13). This large phase III clinical trial, enrolled 931 patients, who were randomly assigned to receive either atezolizumab or second line chemotherapy of investigators' choice. The primary endpoint of overall survival was tested hierarchically in prespecified populations: IC2/3, followed by IC1/2/3, followed by the intention-to-treat population.

The PD-L1 positive population (IC2/3) showed a comparable mOS of 11.1 (95\% CI: 8.6-15.5) months (13) to the phase II data with 11.4 (95\% CI: 9.0-NE) months (24), but the comparator arm showed a mOS of 10.6 (95\% CI: 8.4-12.2) months, which exceeded the study expectations based on the phase III trial evaluating vinflunine compared to BSC $(4,5)$. This resulted in a not significant survival benefit (HR 0.87, 95\% CI: 0.63-1.21; P=0.41).

Though, there are distinct differences between both phase III trials evaluating pembrolizumab $(10,11)$ and atezolizumab (13) compared to second line chemotherapy (Table 1).

\section{Comparison of KEYNOTE-045 and IMvigor211 based on PD-L1 status}

The ORR based on PD-L1 status showed distinct differences between both trials. In KEYNOTE-045, 21.6\% of PD-L1 positive (CPS $\geq 10$ ) and $21.1 \%$ of unselected 
Table 1 Patient characteristics of phase III clinical trials evaluating pembrolizumab and atezolizumab in mUC

\begin{tabular}{|c|c|c|c|c|c|c|}
\hline & \multicolumn{2}{|c|}{$\frac{\text { KEYNOTE-045 }(10,11)}{\text { ITT population }}$} & \multicolumn{4}{|c|}{ IMvigor211 (13) } \\
\hline & $\begin{array}{l}\text { Pembrolizumab } \\
\qquad(n=270)\end{array}$ & $\begin{array}{l}\text { Chemotherapy } \\
\qquad(\mathrm{n}=272)\end{array}$ & $\begin{array}{l}\text { Atezolizumab } \\
\qquad(n=116)\end{array}$ & $\begin{array}{l}\text { Chemotherapy } \\
\qquad(n=118)\end{array}$ & $\begin{array}{l}\text { Atezolizumab } \\
\qquad(\mathrm{n}=467)\end{array}$ & $\begin{array}{l}\text { Chemotherapy } \\
\qquad(\mathrm{n}=464)\end{array}$ \\
\hline Age [range] & 67 [29-88] & 65 [26-84] & 67 [43-88] & 67 [36-84] & 67 [33-88] & 67 [31-84] \\
\hline Upper tract & $14.1 \%$ & $13.7 \%$ & $25.0 \%$ & $22.2 \%$ & $28.7 \%$ & $25.6 \%$ \\
\hline \multicolumn{7}{|l|}{ Metastatic sites } \\
\hline Visceral & $89.3 \%$ & $86.0 \%$ & $67.2 \%$ & $69.5 \%$ & $77.3 \%$ & $76.5 \%$ \\
\hline Positive & $27.4 \%$ & $33.1 \%$ & $100 \%$ & $100 \%$ & $24.8 \%$ & $25.4 \%$ \\
\hline \multicolumn{7}{|l|}{ ECOG performance status } \\
\hline 0 & $44.4 \%$ & $39.0 \%$ & $52.6 \%$ & $48.3 \%$ & $46.7 \%$ & $44.6 \%$ \\
\hline 1 & $52.6 \%$ & $58.1 \%$ & $52.6 \%$ & $51.7 \%$ & $53.3 \%$ & $55.4 \%$ \\
\hline 2 & $1.1 \%$ & $1.5 \%$ & $0.0 \%$ & $0.0 \%$ & $0.0 \%$ & $0.0 \%$ \\
\hline \multicolumn{7}{|l|}{ Number of risk factors } \\
\hline 0 & $20.0 \%$ & $16.5 \%$ & $37.9 \%$ & $34.7 \%$ & $31.0 \%$ & $30.2 \%$ \\
\hline First line & $68.1 \%$ & $58.1 \%$ & $46.6 \%$ & $50.0 \%$ & $53.3 \%$ & $56.3 \%$ \\
\hline Second line & $20.4 \%$ & $21.7 \%$ & $15.5 \%$ & $15.3 \%$ & $16.9 \%$ & $15.9 \%$ \\
\hline Third line & $0.0 \%$ & $0.7 \%$ & $0.9 \%$ & $0.0 \%$ & $1.7 \%$ & $1.9 \%$ \\
\hline Prior radical surgery & $22.6 \%$ & $18.8 \%$ & $49.1 \%$ & $49.2 \%$ & $42.6 \%$ & $43.1 \%$ \\
\hline \multicolumn{7}{|c|}{ Chemotherapy on trial (safety population) } \\
\hline Vinflunine & - & $34.1 \%(87 / 255)$ & - & n.a. & - & $54.6 \%(242 / 443)$ \\
\hline Docetaxel & - & $32.9 \%(84 / 255)$ & - & n.a. & - & $12.0 \%(53 / 443)$ \\
\hline Paclitaxel & - & $32.9 \%(84 / 255)$ & - & n.a. & - & $33.4 \%(148 / 443)$ \\
\hline
\end{tabular}

Table 1 (Continued) 
Table 1 (Continued)

\begin{tabular}{|c|c|c|c|c|c|c|}
\hline & \multicolumn{2}{|c|}{$\frac{\text { KEYNOTE-045 }(10,11)}{\text { ITT population }}$} & \multicolumn{4}{|c|}{ IMvigor211 (13) } \\
\hline & $\begin{array}{l}\text { Pembrolizumab } \\
\qquad(n=270)\end{array}$ & $\begin{array}{l}\text { Chemotherapy } \\
\qquad(\mathrm{n}=272)\end{array}$ & $\begin{array}{l}\text { Atezolizumab } \\
\qquad(\mathrm{n}=116)\end{array}$ & $\begin{array}{l}\text { Chemotherapy } \\
\qquad(\mathrm{n}=118)\end{array}$ & $\begin{array}{l}\text { Atezolizumab } \\
\qquad(\mathrm{n}=467)\end{array}$ & $\begin{array}{c}\text { Chemotherapy } \\
(n=464)\end{array}$ \\
\hline $\begin{array}{l}\text { Subsequent treatment (ITT } \\
\text { population) }\end{array}$ & $25.2 \%$ & $33.5 \%$ & n.a. & n.a. & $23.1 \%$ & $25.4 \%$ \\
\hline Immunotherapy & n.a. & n.a. & n.a. & n.a. & $1.3 \%$ & $6.0 \%$ \\
\hline Others & n.a. & n.a. & n.a. & n.a. & $4.5 \%$ & $1.7 \%$ \\
\hline
\end{tabular}

ITT, intention-to-treat; n.a., not available.

patients responded to pembrolizumab, while the ORR to chemotherapy was lower with $11.4 \%$ and $6.7 \%$ in the total population and the CPS $\geq 10$ intention-to-treat (ITT) population, respectively (10). Opposingly, response rates to atezolizumab were higher in the IC2/3 (IC $\geq 5 \%$ ) population than in the unselected ITT population in IMvigor2 11 (23.0\% vs. 13.4\%) (13). Interestingly, also the response to chemotherapy was better in patients with $\mathrm{PD}-\mathrm{L} 1$ positive tumors compared to unselected patients $(21.6 \% \mathrm{vs}$. $13.4 \%$ ) (13). Unexpectedly, overexpression of PD-L1 resulted in a more favorable outcome with increased ORR and longer overall survival for both, chemotherapy and atezolizumab in IMvigor211. This negates the potential predictive value of PD-L1, when stained with SP142 and analyzed for tumor infiltrating IC, and suggest a rather prognostic effect of PD-L1 in this setting (13).

In KEYNOTE-045 a significantly longer mOS for pembrolizumab over chemotherapy has been observed in unselected patients (10.1 vs. 7.3 months; $95 \%$ CI: $0.57-$ 0.85; HR 0.70) and patients harboring PD-L1 positive tumors $(\mathrm{CPS} \geq 10$ ) with 8.0 vs. 4.9 months $(\mathrm{P}=0.00122)$, respectively (11). Remarkable, in KEYNOTE-045 PD-L1 expression (antibody 22C3; CPS $\geq 10$ ) seems to have a negative prognostic effect, while in IMvigor211 (antibody SP142; IC $\geq 5 \%$ ) in seems to have positive prognostic properties.

\section{Comparison of KEYNOTE-045 and IMvigor211 based on second line chemotherapeutic agents}

In KEYNOTE-045, pembrolizumab showed a mOS 10.1 vs. 7.4 months in unselected patients for PD-L1 status and compared to second line chemotherapy of investigators' choice (95\% CI: 6.1-8.3) (10). Exploratory analysis of the IMvigor2 11 trial revealed a mOS of 8.6 (95\% CI: 7.8-9.6) months for atezolizumab and 8.0 (95\% CI: 7.2-8.6) months for second line chemotherapy in the ITT population (13). Further analysis, stratified by the choice of second line chemotherapy, showed a survival benefit of atezolizumab compared to taxane-based chemotherapy (docetaxel, paclitaxel) with a mOS of $8.3 v s .7 .5$ months (HR 0.73; $95 \%$ CI: 0.58-0.92), but not over vinflunine with a mOS of 9.2 vs. 8.3 months (HR 0.97; 95\% CI: 0.78-1.19) (25).

One of the major differences between both trials is the distribution of chemotherapeutic agents in the comparator arms. In IMvigor 211, the majority of patients in the chemotherapy arm $(\mathrm{n}=443)$ received vinflunine $(55 \%)$, while paclitaxel (33\%) and docetaxel (12\%) where less frequently chosen by investigators in this trial (13). This might be related to the study population, with the majority of patients being recruited in Western Europe, where vinflunine is an approved second line chemotherapeutic agent $(4,5)$. In KEYNOTE-045, distribution of chemotherapeutic agents was well balanced in the chemotherapy arm $(10,11)$.

\section{Comparison of KEYNOTE-045 and IMvigor211 based on primary tumor and metastatic site}

IMvigor211 included more upper tract urothelial tumors (UTUC) than KEYNOTE-045, which were evenly distributed between the treatment arms. While $25.3 \%$ of included patients in IMvigor2 11 had UTUC, only $13.8 \%$ of patients in KEYNOTE-045 harboring UTUCs $(11,13)$.

Rates of patients with visceral and liver metastases were higher in KEYNOTE-045 with $87.8 \%$ and $34.3 \%$ (11) compared to IMvigor 211 with $68.4 \%$ and $24.8 \%$ in the 

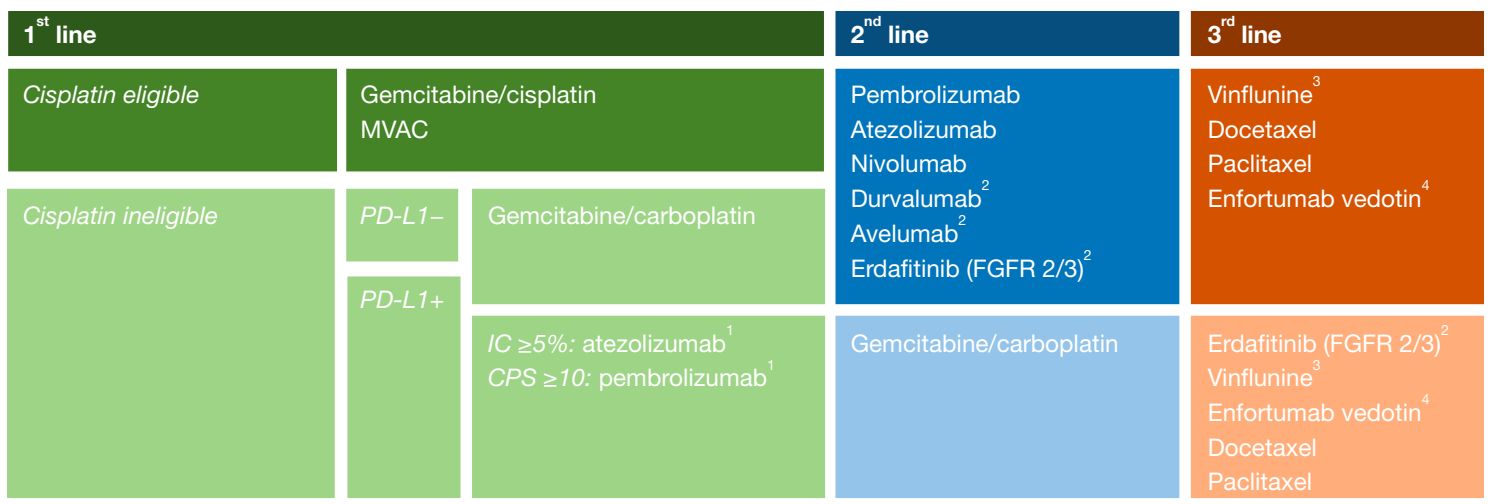

Figure 1 Proposed treatment algorithm based on current evidence from clinical trials. ${ }^{1}$, FDA approved in platin ineligible pts irrespective of PD-L1 status; ${ }^{2}$, FDA approved only; ${ }^{3}$, EMA, approved only; ${ }^{4}$, awaiting approval.

IC2/3 and $76.9 \%$ and $28.8 \%$ in the ITT population (13). Patients with PD-L1 positive tumors (IC2/3) and liver metastasis showed a superior response to atezolizumab compared to chemotherapy in IMvigor2 11 (HR 0.51; 95\% CI: 0.28-0.96). Rates of patients with lymph nodes (LN) only disease were comparable between both trials with $12.9 \%$ (IMvigor211) and $12.0 \%$ (KEYNOTE-045) $(11,13)$. Though, the chemotherapy arm in IMvigor211 (IC2/3 population) included a higher rate of patients with $\mathrm{LN}$ only disease (22.9\%) compared to only $15.5 \%$ in the PD-L1 positive population treated with atezolizumab (13). Nevertheless, this factor cannot explain the good response to chemotherapy in this trial, since LN only patients show favorable response to atezolizumab compared to chemotherapy in patients with $\mathrm{PD}-\mathrm{L} 1$ positive tumors (HR 0.60, 95\% CI: 0.26-1.36) and even better in the unselected ITT population (HR 0.59; 95\% CI: 0.37-0.96).

\section{Comparison of KEYNOTE-045 and IMvigor211 based on risk factors and previous and subsequent off-study treatments}

IMvigor211 and KEYNOTE-045 recruited different baseline population with lower rates of patients without any Bajorin risk factors in IMvigor211 (10,13). In addition, more patients underwent prior surgery of the primary tumor in IMvigor211 than in KEYNOTE-045, with 42.9\% vs. $20.7 \%$, respectively $(10,13)$. This raises the question of whether presence of the primary tumor plays a role in response to CPI in $\mathrm{mUC}$.

The higher rate of primary tumor resection also explains the higher rate of patients, who underwent previous neoadjuvant and adjuvant chemotherapy in IMvigor211 (24.2\% vs. $15.5 \%)$ compared to KEYNOTE-045 $(10,13)$. This rate was even higher in the IC2/3 population of IMvigor 211 with $31.6 \%$ of patients (13). In both phase III trials the rate of subsequent treatments was relatively high, with more than $20 \%$ of patients having received following treatments after on study treatment $(10,11,13)$, which reflects the increasing therapeutic options for patients with mUC (Figure 1).

Both trials have evaluated a CPI in second line after failure of platinum-based chemotherapy. Though, comparison and interpretation of differences between those trials is difficult. These two, phase III trials studied distinct different patient populations, PD-L1 has been evaluated with different antibodies, staining platforms and scoring methods, and the comparator arms had a different distribution of second line chemotherapeutic agents $(10,11,13)$. In addition, IMvigor211 used a biomarker driven design, using PD-L1 positivity in a hierarchal statistical design of several subgroup analysis (13). This was based on promising results from earlier phase clinical trials, where PD-L1 seemed to be a predictive marker of response to atezolizumab in this setting (24). All these factors might have influenced the outcome and failure of IMvigor211 to proof superiority of, the clinically active substance, atezolizumab over second line chemotherapy in a phase III trial.

Nevertheless, pembrolizumab is the only CPI with level 1 evidence of superiority over chemotherapy in second line treatment of mUC as proven in KEYNOTE-045 so far $(10,11)$. The rapidly developing treatment landscape giving hope for our patients suffering from UC and urges the necessity to develop a biomarker driven clinical decision- 
making strategy, since by far not all patients benefit from the available therapeutic options.

\section{Acknowledgments}

None.

\section{Footnote}

Conflicts of Interest: KM Gust has received advisory board fees from Cepheid, Roche, MSD, and Ferring; speaker fees from Amgen, Astellas, AstraZeneca, BMS, Ipsen, Janssen, MSD, and Roche; and meeting/travel expenses from Allergan, Astellas, AstraZeneca, Bayer, BMS, Janssen, MSD, Novartis, Pfizer, Pierre Fabre, and Roche. SF Shariat has received advisory board and/or speaker fees from Astellas, AstraZeneca, Bayer, BMS, Cepheid, Ferring, Ipsen, Janssen, Lilly, MSD, Olympus, Pfizer, Pierre Fabre, Roche, Sanochemia, Sanofi, UroGen, and Wolff. The other authors have no conflicts of interest to declare.

Ethical Statement: The authors are accountable for all aspects of the work in ensuring that questions related to the accuracy or integrity of any part of the work are appropriately investigated and resolved.

\section{References}

1. Hautmann RE, de Petriconi RC, Pfeiffer C, et al. Radical cystectomy for urothelial carcinoma of the bladder without neoadjuvant or adjuvant therapy: long-term results in 1100 patients. Eur Urol 2012;61:1039-47.

2. Sternberg CN, de Mulder PH, Schornagel JH, et al. Randomized phase III trial of high-dose-intensity methotrexate, vinblastine, doxorubicin, and cisplatin (MVAC) chemotherapy and recombinant human granulocyte colony-stimulating factor versus classic MVAC in advanced urothelial tract tumors: European Organization for Research and Treatment of Cancer Protocol no. 30924. J Clin Oncol 2001;19:2638-46.

3. von der Maase H, Sengelov L, Roberts JT, et al. Longterm survival results of a randomized trial comparing gemcitabine plus cisplatin, with methotrexate, vinblastine, doxorubicin, plus cisplatin in patients with bladder cancer. J Clin Oncol 2005;23:4602-8.

4. Bellmunt J, Theodore C, Demkov T, et al. Phase III trial of vinflunine plus best supportive care compared with best supportive care alone after a platinum-containing regimen in patients with advanced transitional cell carcinoma of the urothelial tract. J Clin Oncol 2009;27:4454-61.

5. Bellmunt J, Fougeray R, Rosenberg JE, et al. Longterm survival results of a randomized phase III trial of vinflunine plus best supportive care versus best supportive care alone in advanced urothelial carcinoma patients after failure of platinum-based chemotherapy. Ann Oncol 2013;24:1466-72.

6. Necchi A, Pond GR, Raggi D, et al. Efficacy and Safety of Gemcitabine Plus Either Taxane or Carboplatin in the First-Line Setting of Metastatic Urothelial Carcinoma: A Systematic Review and Meta-Analysis. Clin Genitourin Cancer 2017;15:23-30.e2.

7. Shariat SF, Gust KM. Immune therapy meets precision medicine. Lancet Oncol 2017;18:271-3.

8. Balar AV, Castellano DE, O'Donnell PH, et al. Pembrolizumab as first-line therapy in cisplatinineligible advanced urothelial cancer: Results from the total KEYNOTE-052 study population. J Clin Oncol 2017;35:abstr 284.

9. Balar AV, Galsky MD, Rosenberg JE, et al. Atezolizumab as first-line treatment in cisplatin-ineligible patients with locally advanced and metastatic urothelial carcinoma: a single-arm, multicentre, phase 2 trial. Lancet 2017;389:67-76.

10. Bellmunt J, de Wit R, Vaughn DJ, et al. Pembrolizumab as Second-Line Therapy for Advanced Urothelial Carcinoma. N Engl J Med 2017;376:1015-26.

11. Fradet Y, Bellmunt J, Vaughn DJ, et al. Randomized phase III KEYNOTE-045 trial of pembrolizumab versus paclitaxel, docetaxel, or vinflunine in recurrent advanced urothelial cancer: results of $>2$ years of follow-up. Ann Oncol 2019. [Epub ahead of print].

12. Patel MR, Ellerton J, Infante JR, et al. Avelumab in metastatic urothelial carcinoma after platinum failure (JAVELIN Solid Tumor): pooled results from two expansion cohorts of an open-label, phase 1 trial. Lancet Oncol 2018;19:51-64.

13. Powles T, Duran I, van der Heijden MS, et al. Atezolizumab versus chemotherapy in patients with platinum-treated locally advanced or metastatic urothelial carcinoma (IMvigor211): a multicentre, open-label, phase 3 randomised controlled trial. Lancet 2018;391:748-57.

14. Powles T, O'Donnell PH, Massard C, et al. Efficacy and Safety of Durvalumab in Locally Advanced or Metastatic Urothelial Carcinoma: Updated Results From a Phase 1/2 Open-label Study. JAMA Oncol 2017;3:e172411. 
15. Sharma P, Retz M, Siefker-Radtke A, et al. Nivolumab in metastatic urothelial carcinoma after platinum therapy (CheckMate 275): a multicentre, single-arm, phase 2 trial. Lancet Oncol 2017;18:312-22.

16. Loriot Y, Necchi A, Park SH, et al. Erdafitinib in Locally Advanced or Metastatic Urothelial Carcinoma. N Engl J Med 2019;381:338-48.

17. Rosenberg JE, O'Donnell PH, Balar AV, et al. Pivotal Trial of Enfortumab Vedotin in Urothelial Carcinoma After Platinum and Anti-Programmed Death 1/Programmed Death Ligand 1 Therapy. J Clin Oncol 2019;37:2592-600.

18. Necchi A, Anichini A, Raggi D, et al. Pembrolizumab as Neoadjuvant Therapy Before Radical Cystectomy in Patients With Muscle-Invasive Urothelial Bladder Carcinoma (PURE-01): An Open-Label, Single-Arm, Phase II Study. J Clin Oncol 2018:JCO1801148. [Epub ahead of print]

19. Powles T, Rodriguez-Vida A, Duran I, et al. A phase II study investigating the safety and efficacy of neoadjuvant atezolizumab in muscle invasive bladder cancer (ABACUS). J Clin Oncol 2018;36:abstr 4506.

20. Gust KM, Shariat SF. Re: Pembrolizumab as Neoadjuvant Therapy Before Radical Cystectomy in Patients with Muscle-invasive Urothelial Bladder Carcinoma (PURE-

Cite this article as: Gust KM, Abufaraj M, D'Andrea D, Moschini M, Soria F, Shariat SF. Second line immune checkpoint inhibition in urothelial cancer. Transl Androl Urol 2019;8(5):414-420. doi: 10.21037/tau.2019.09.18
01): An Open-label, Single-arm, Phase II Study. Eur Urol 2019;75:695-6.

21. Galsky MD, Hahn NM, Rosenberg J, et al. Treatment of patients with metastatic urothelial cancer "unfit" for Cisplatin-based chemotherapy. J Clin Oncol 2011;29:2432-8.

22. FDA. Available online: https://www.fda.gov/drugs/ drugsafety/ucm608075.htm, 2018.

23. EMA. EMA/364553/2018. Available online: https://www. ema.europa.eu/en/documents/press-release/ema-restrictsuse-keytruda-tecentriq-bladder-cancer_en.pdf, 2018.

24. Rosenberg JE, Hoffman-Censits J, Powles T, et al. Atezolizumab in patients with locally advanced and metastatic urothelial carcinoma who have progressed following treatment with platinum-based chemotherapy: a single-arm, multicentre, phase 2 trial. Lancet 2016;387:1909-20.

25. Powles T, Loriot Y, Ravaud A, et al. Atezolizumab vs Chemotherapy in Platinum-Treated Locally Advanced or Metastatic Urothelial Carcinoma: Immune Biomarker, Tumor Mutational Burden and Clinical Outcomes From the Phase III IMvigor 211 Study. J Clin Oncol 2018;36:abstr 409. 